\title{
RESEARCH
}

Open Access

\section{Haemoglobin, magnetic resonance imaging markers and cognition: a subsample of population-based study}

\author{
Bryce Tan ${ }^{1}$, Narayanaswamy Venketasubramanian², Henri Vrooman ${ }^{3}$, Ching-Yu Cheng ${ }^{4,5}$, Tien Yin Wong ${ }^{4,5}$,
} Christopher Chen ${ }^{1,6}$ and Saima Hilal ${ }^{1,6,7^{*}}$

\begin{abstract}
Background: Low haemoglobin is highly prevalent among the elderly and has been associated with dementia. However, the mechanisms underlying this association with cognitive dysfunction, either through cerebrovascular disease or neurodegeneration, remain poorly understood. We aimed to examine the association of decreased haemoglobin levels with markers of cerebral small vessel disease (CSVD), neurodegeneration and cognitive impairment in an elderly Asian population.

Methods: A total of 796 Chinese, Malay and Indian participants aged 60 years and older from the Epidemiology of Dementia in Singapore study were included in this study. After providing information on demographics, anthropometry and cardiovascular risk factors, participants underwent 3-T brain magnetic resonance imaging (MRI) to measure markers of CSVD, including cerebral microbleeds, cortical cerebral microinfarcts, lacunes, enlarged perivascular spaces and white matter hyperintensities, as well as neurodegenerative markers, including cortical thickness and subcortical structure volumes quantified using FreeSurfer. Cognition was assessed using a detailed neuropsychological assessment. Logistic and linear regression models were constructed, adjusting for age, gender, education, race, body mass index, smoking, hypertension, hyperlipidaemia, diabetes, glomerular filtration rate and other MRI markers, to test the association between haemoglobin levels and the MRI markers and cognition.
\end{abstract}

Results: Decreased haemoglobin levels were associated with cerebral microbleeds, specifically lobar microbleeds $(\mathrm{OR}, 1.21 ; 95 \% \mathrm{Cl}, 1.04-1.40 ; p=0.015)$. Decreased haemoglobin levels were also associated with occipital cortical thinning (mean difference, $-0.011 ; 95 \% \mathrm{Cl},-0.019,-0.004 ; p=0.003$ ) and smaller accumbens volume (mean difference, -0.01 ; $95 \% \mathrm{Cl},-0.02,0.00 ; p=0.005$ ). A significant association was also observed between decreased haemoglobin levels and poorer global cognitive performance (mean difference, $-0.04 ; 95 \% \mathrm{Cl},-0.09,0.00 ; p=0.048$ ). In cognitive domain analysis, associations were again observed between decreased haemoglobin levels and worse performance on attention (mean difference, $-0.05 ; 95 \% \mathrm{Cl},-0.10,-0.01 ; p=0.028$ ) and language (mean difference, $-0.06 ; 95 \% \mathrm{Cl},-0.12,0.00 ; p=0.048)$ domains; however, these associations did not survive multiple comparison.

(Continued on next page)

\footnotetext{
* Correspondence: phchs@nus.edu.sg

${ }^{1}$ Memory Ageing and Cognition Centre (MACC), National University Health System, Singapore, Singapore

${ }^{6}$ Department of Pharmacology, Yong Loo Lin School of Medicine, National University of Singapore, Level 4, Block MD3, 16 Medical Drive, Singapore 117600, Singapore

Full list of author information is available at the end of the article
}

(c) The Author(s). 2018 Open Access This article is distributed under the terms of the Creative Commons Attribution 4.0 International License (http://creativecommons.org/licenses/by/4.0/), which permits unrestricted use, distribution, and reproduction in any medium, provided you give appropriate credit to the original author(s) and the source, provide a link to the Creative Commons license, and indicate if changes were made. The Creative Commons Public Domain Dedication waiver (http://creativecommons.org/publicdomain/zero/1.0/) applies to the data made available in this article, unless otherwise stated. 
(Continued from previous page)

Conclusions: Decreased haemoglobin levels were associated with lobar microbleeds, neurodegenerative markers and cognitive dysfunction. Future studies should ascertain whether iron, folate or vitamin $B_{12}$ supplementation is able to ameliorate the onset and progression of cognitive impairment and dementia associated with low haemoglobin.

Keywords: Haemoglobin, Anaemia, Cortical thinning, Microbleed, Cognitive impairment

\section{Background}

There is growing evidence that anaemia is a common condition in the elderly; it has a prevalence exceeding $10 \%$ in people aged 65 years or older [1]. About one-third of anaemia diagnoses in the elderly are attributed to nutritional deficits which are easily treatable by adequate nutritional support with iron, vitamin $B_{12}$ and folate supplements [2]. Recently, it has been suggested that even modest decreases in haemoglobin concentrations among those not classified as anaemic are associated with increased morbidity and mortality [3-5]. Although previous studies have shown that decreased haemoglobin levels are associated with cognitive impairment [6] and risk of dementia [7-9], these associations are inconsistent [10].

Non-invasive magnetic resonance imaging (MRI) markers of cerebral small vessel disease (CSVD) include lacunes, white matter hyperintensities, cerebral microbleeds and cortical cerebral microinfarcts, whereas surrogate markers of neurodegeneration include cortical and subcortical atrophy. Limited data has shown that anaemia is associated with a chronic hypoxic state that contributes to increased cerebrovascular burden, resulting in increased white matter hyperintensity volume [11-13]. However, a recent study showed no association between lower haemoglobin levels and the presence or progression of CSVD [14]. Moreover, the association of haemoglobin with cerebral microbleeds, and cortical cerebral microinfarcts remain unstudied. With respect to neurodegenerative markers, only one study reported that lower haemoglobin levels are linked to cortical thinning among cognitively normal women [14]. These differences in results may be attributed to heterogeneity in defining anaemia (binary criteria vs. percentiles) or small sample sizes, or they may be related to the study of particular disease populations (dementia, chronic kidney disease and hypertension). Hence studies from a general population are needed to investigate the relationship between decreased haemoglobin levels and markers of CSVD and neurodegeneration to investigate the underlying mechanisms. Such findings would have potential clinical significance for preventing cognitive impairment and improving cognitive function in the elderly.

We aimed to examine the association of haemoglobin with markers of CSVD (cerebral microbleeds, cortical cerebral microinfarcts, lacunes, enlarged perivascular spaces and white matter hyperintensities) and neurodegeneration (cortical thickness and subcortical structure volume). We also examined the effects of decreased haemoglobin levels on cognitive performance in a multi-ethnic Asian population. We hypothesize that decreased haemoglobin levels induce a hypoxic state leading to ischemia as well as atrophy in subcortical and cortical regions.

\section{Methods \\ Study population}

The Epidemiology of Dementia in Singapore study recruited individuals from the Singapore Epidemiology of Eye Disease study, which comprised participants between 40 and 85 years old who participated in the Singapore Chinese Eye Study, the Singapore Malay Eye Study and the Singapore Indian Eye Study [15]. Briefly, participants aged 60 years and older were screened with the Abbreviated Mental Test (AMT) and a self-reported history of forgetfulness. Screen-positives were defined on the basis of education-based cut-offs on AMT (AMT $\leq 6$ in subjects with $\leq 6$ years of formal education or $\leq 8$ among those with $>6$ years of formal education) or if the caregiver confirmed progressive forgetfulness. Thus the inclusion criteria of the study included (1) screen-positive on AMT or Present Functioning Questionnaire and (2) written informed consent given by participants or their legally acceptable representatives. Of these 1598 screen-positive participants, 957 agreed to participate in the second phase, which included brain MRI and extensive cognitive assessment. Exclusion criteria included participant or legally acceptable representative not willing to provide written informed consent. Ethics approval was obtained from the Singapore Eye Research Institute and the National Healthcare Group domain-specific review board, and written consent was obtained from participants.

\section{Blood tests}

Participants were required to fast for at least $8 \mathrm{~h}$ prior to blood tests. Fasting blood samples were sent to the National University Hospital Laboratory for measurements of the following: full blood count, glucose, lipid panel and creatinine. Haemoglobin was measured with the 
Sysmex XN-Series automated haematology analyser 2012 version (Streck, Omaha, NE, USA). The lipid panel comprised total cholesterol, low-density lipoprotein, high-density lipoprotein and triglyceride levels.

\section{Neuroimaging}

MRI scans were obtained using a 3-T MAGNETOM Trio Tim scanner (Siemens, Erlangen, Germany) with a 32-channel head coil at the Clinical Imaging Research Centre, National University of Singapore. Subjects with claustrophobia, with contraindications for MRI, or who were unable to tolerate the procedure were excluded. STRIVE (Standards for Reporting Vascular Changes on Neuroimaging) criteria were used for grading of lacunes on fluid-attenuated inversion recovery (FLAIR) and T2-weighted sequences [16]. Cortical cerebral microinfarcts were graded on FLAIR, T1- and T2-weighted images and were defined as hypointense lesions on T1-weighted images, restricted to the cortex, $<5 \mathrm{~mm}$ in diameter, and perpendicular to the cortical surface. These lesions were further confirmed as hyperintense or isointense on T2-weighted and FLAIR images as previously described [17] and were analysed as a categorical variable. Cerebral microbleeds were graded using the Brain Observer Microbleed Scale [18] and are classified as lobar or deep microbleeds as dichotomous variables. Enlarged perivascular spaces were categorized as $\leq 10$ vs. $\geq 11$ to compare moderate to severe enlarged perivascular spaces with mild to absent perivascular spaces [19].

Total intracranial volume was quantified using T1- and T2-weighted images, whereas white matter hyperintensity volume was quantified with FLAIR images using automatic segmentation at the Erasmus University Medical Centre, the Netherlands. A model-based automated procedure (FreeSurfer version 5.1.0) was used for measurements of cortical thickness and subcortical structure volumes on T1-weighted images. Briefly, cortical thickness was measured by taking the shortest distance between the whitegrey matter boundary and pial surface for regional lobes and the whole brain at each vertex. Cortical thickness of the frontal, insular, limbic, occipital, parietal and temporal regions was measured, and a parcellation guide on the gyral and sulcal structures of the cerebral cortex was used in calculating the average cortical thickness of the left and right lobes [20]. Volumes of subcortical structures of each hemisphere (accumbens, amygdala, brainstem, caudate, hippocampus, putamen, pallidum and thalamus) were calculated by segmentation using rigid-body registration and subjected to non-linear normalization with respect to a probabilistic brain atlas [21].

\section{Covariate assessment}

Demographic and cardiovascular risk factors were collected during the interview and included age, gender, education, smoking history, height, weight, hypertension, hyperlipidaemia and diabetes mellitus. A digital automatic blood pressure machine (HEM-7203; OMRON, Kyoto, Japan) was used for measurement of two systolic and diastolic blood pressure readings taken 5 min apart after the participant had rested for $5 \mathrm{~min}$. Hypertension was defined as the use of anti-hypertensive medication or systolic blood pressure $\geq 140 \mathrm{mmHg}$ and/or diastolic blood pressure $\geq 90 \mathrm{mmHg}$. Diabetes mellitus was defined as the use of diabetic medications or a glycosylated haemoglobin $\geq 6.5 \%$. Hyperlipidaemia was defined as the use of lipid-lowering medications or total cholesterol level $\geq 4.14 \mathrm{mmol} / \mathrm{L}$. Education was categorized as $\leq$ 6 years or $>6$ years of formal education. Body mass index (BMI) was calculated by weight in kilograms divided by height in meters squared. Smoking was categorized into ever vs. never smokers. Socio-economic status was defined by monthly income and housing. A low socio-economic status was categorized by monthly income $<2000$ Singapore dollars per household and living in $\leq 2$-room public housing flats.

\section{Cognitive assessment}

All participants underwent detailed cognitive assessment using validated methods [15]. The following seven domains were tested: attention, executive function, language, visual memory, verbal memory, visuomotor speed and visuoconstruction. Each domain was tested using the following cognitive tests:

- Attention: Digit Span, Visual Memory Span [22] and Auditory Detection [23]

- Executive function: Frontal Assessment Battery [24] and Maze Task [25]

- Language: Boston Naming Test [26] and Verbal Fluency [27]

- Verbal memory: Word List Recall [28] and Story Recall

- Visual memory: Picture Recall, Wechsler Memory Scale-Revised (WMS-R) Visual Reproduction [22]

- Visuoconstruction: WMS-R Visual Reproduction Copy Task [22], Clock Drawing [29], and Wechsler Adult Intelligence Scale-Revised subtest of block design [30]

- Visuomotor speed: Symbol Digit Modality Test [31] and Digit Cancellation [32].

For each participant, raw scores from each individual test within a domain were first transformed to standardized Z-scores using the mean and SD of that test in this cohort. Subsequently, for each participant a mean Z-score for each domain was calculated by averaging the Z-scores of all the individual tests within that domain. These mean Z-scores of each domain were then standardized using the 
mean and SD of that domain-specific Z-score. Finally, a composite Z-score reflecting global cognitive functioning was calculated by averaging the seven domain-specific mean Z-scores, which were also standardized using the corresponding mean and SD.

\section{Statistical Analysis}

Comparisons between included and excluded participants were performed using the chi-square test for categorical variables and Student's $t$ test for continuous variables. Binary logistic regression models with ORs and 95\% CIs were constructed to determine the association of haemoglobin with microbleeds, cortical cerebral microinfarcts, lacunes and enlarged perivascular spaces, whereas linear regression models were used to determine the association between haemoglobin and white matter hyperintensity volumes with mean differences and $95 \%$ CIs. Linear regression models were again constructed to determine the association of haemoglobin with global and regional cortical thickness, subcortical structural volume, and cognitive domains. Models were initially adjusted for age, gender, race, smoking status and education (in analysis with cognition) (model I). Further adjustments were made for vascular risk factors, which included diabetes mellitus, hyperlipidaemia, hypertension, glomerular filtration rate and BMI (model II). In the fully adjusted model (model III), socio-economic status (in analysis with cognition) and MRI markers (microbleeds, cortical cerebral microinfarcts, lacunes, enlarged perivascular spaces, white matter hyperintensity volume, microbleeds) were included to investigate the independent effects of haemoglobin on cortical thickness, subcortical structure volume, and cognition. Mean differences or ORs, 95\% CIs and their corresponding $p$ values in the tables correspond to the effect estimate for each gram per decilitre drop in haemoglobin. $p$ values $<0.05$ were considered significant. In view of the multiple testing performed in cortical thickness, subcortical structures and cognition models, the Bonferroni correction was applied with the significance levels set at $p=0.025(0.05 / 2)$ for CSVD, $p=0.0083(0.05 /$ 6) for cortical thickness, $p=0.00625(0.05 / 8)$ for subcortical structures and $p=0.0071(0.05 / 7)$ for cognition. All statistical analyses were performed using standard statistical software (IBM SPSS Statistics version 24; IBM, Armonk, NY, USA).

\section{Results}

Assessment of subjects was performed from August 12, 2010, to July 24, 2015. Among 957 subjects, 46 were diagnosed with dementia, 96 had ungradable MRI scans and 19 had no haemoglobin results available. The final sample consisted of 796 subjects. Table 1 presents the baseline data of the included $(n=796)$ and excluded $(n=756)$ subjects. The excluded group consisted of positive-screened non-responders, persons with ungradable MRI scans or absent haemoglobin levels. Briefly, excluded subjects were older, more likely to be female, to be less educated and to have hypertension and less likely to have hyperlipidaemia.

Table 2 presents the association of haemoglobin with MRI markers of CSVD. Decreased levels of haemoglobin were associated with cerebral microbleeds (OR, 1.16; 95\% CI, 1.01-1.33), specifically those located in the lobar region (OR, 1.21; 95\% CI, 1.04-1.40) in the multivariable model (even after adjusting for multiple comparisons). Although decreased levels of haemoglobin were associated with lacunes (OR, 1.25; 95\% CI, 1.07-1.46) in the models adjusted for age, gender, race and smoking, the association became attenuated in the presence of cardiovascular risk factors and other MRI markers of CSVD.

Table 3 presents the associations of haemoglobin with global and regional cortical thickness. Decreased haemoglobin levels were associated with smaller global (mean difference, $-0.006,95 \%$ CI: $-0.013 ;-0.001)$ and occipital (mean difference, -0.012 ; 95\% CI, $-0.020,-0.005$ ) cortical thickness after controlling for demographic and cardiovascular risk factors. These associations remained significant after additional adjustment for markers of CSVD and intracranial volume. When we applied the Bonferroni correction, decreased haemoglobin levels remained significantly linked with occipital cortical thinning. With respect to the subcortical structures, decreased levels of haemoglobin were associated with smaller accumbens volume (mean difference, - 0.02; $95 \% \mathrm{CI},-0.03,-0.01)$ in the demographic and cardiovascular risk factors model. These associations remained unaltered after controlling for MRI markers and survived multiple testing. No association was observed with other subcortical structure volumes (Table 4).

Decreased levels of haemoglobin were also significantly associated with poor cognitive performance (mean difference in global cognitive z-scores, - 0.06; 95\% CI, $0.10,-0.01)$ as well as with individual cognitive domains of attention (mean difference, - 0.06; 95\% CI, - 0.11, 0.01 ) and language (mean difference, -0.07 ; $95 \% \mathrm{CI}$, $0.13,-0.02$ ) after adjustment for demographic and cardiovascular risk factors. Adjustment for socio-economic status and other MRI markers of CSVD rendered similar results, suggesting that the associations of haemoglobin with cognition were not mediated by these variables (Table 5). However, these associations did not reach the revised level of significance after the Bonferroni correction was applied.

\section{Discussion}

Our results showed that decreased levels of haemoglobin were associated with lobar microbleeds, global and occipital cortical thinning, and smaller accumbens volume independent of cardiovascular risk factors and other MRI 
Table 1 Baseline characteristics of included and excluded subjects

\begin{tabular}{|c|c|c|c|}
\hline & Included $(n=796)$ & Excluded $(n=756)^{\mathrm{a}}$ & $p$ Value \\
\hline Age, years, mean (SD) & $70.0(6.6)$ & $71.9(6.9)$ & $<0.001$ \\
\hline Female sex, $n(\%)$ & $432(54.3)$ & $433(57.3)$ & 0.015 \\
\hline Education ( $\leq 6$ years), $n(\%)$ & $489(61.4)$ & $554(73.3)$ & $<0.001$ \\
\hline Ethnicity & & & $<0.001$ \\
\hline Chinese, $n(\%)$ & $273(34.2)$ & $333(44.0)$ & \\
\hline Malay, $n$ (\%) & $263(33.0)$ & $194(25.7)$ & \\
\hline Indian, $n(\%)$ & $260(32.7)$ & $229(30.3)$ & \\
\hline Diabetes mellitus, $n$ (\%) & $289(36.3)$ & $260(34.4)$ & 0.430 \\
\hline Hypertension, $n$ (\%) & $621(78.0)$ & $634(83.9)$ & 0.003 \\
\hline Hyperlipidaemia, $n(\%)$ & $586(73.6)$ & $498(65.9)$ & 0.001 \\
\hline Smoking, $n(\%)$ & $198(24.9)$ & $185(24.4)$ & 0.854 \\
\hline $\mathrm{BMl}, \mathrm{kg} / \mathrm{m}^{2}$, mean (SD) & $23.4(4.6)$ & $23.6(4.6)$ & 0.982 \\
\hline Mean arterial blood pressure, mmHg, mean (SD) & $97.4(10.4)$ & $97.8(11.3)$ & 0.282 \\
\hline Total cholesterol, mmol/L, mean (SD) & $5.1(1.2)$ & $5.2(1.2)$ & 0.354 \\
\hline Random blood glucose, mmol/L, mean (SD) & $7.1(3.1)$ & $7.1(3.1)$ & 0.846 \\
\hline Haemoglobin, g/dl, mean (SD) & $13.4(1.4)$ & - & - \\
\hline Creatinine, $\mu \mathrm{mol} / \mathrm{L}$, median (IQR) & $72(30)$ & - & - \\
\hline eGFR, ml/min, mean (SD) & $91(29)$ & - & - \\
\hline
\end{tabular}

BMI Body mass index, eGFR Estimated glomerular filtration rate

${ }^{a}$ Excluded subjects were screened positive non-responders, had ungradable magnetic resonance imaging scans or absent haemoglobin levels

markers. Moreover, people with decreased haemoglobin levels had worse cognition specifically in the domains of attention and language, albeit these results were non-significant after correcting for multiple comparisons.

Few studies have explored the link between decreased haemoglobin levels and neuroimaging markers of CSVD, and the results remain controversial. One study has reported a link between anaemia and white matter hyperintensity progression among patients with hypertension [13], whereas a recent study failed to find an association with white matter hyperintensity volume in a large community-based study [14]. The latter study also did not find any significant link with lacunes and cerebral microbleeds [14]. Although we observed a significant association with lacunes in the initial model, the association was attenuated after adjustment for other markers of CSVD. Similarly, we did not observe any significant association with white matter hyperintensity volumes, which is consistent with the previous data. To date, this is the first study exploring the association between haemoglobin and enlarged perivascular spaces. Consistent with lacunes and white matter hyperintensities, we also did not observe an association with enlarged perivascular spaces, suggesting a similar underlying mechanism for the development of these lesions. On the contrary, we report an association of decreased haemoglobin levels with cerebral microbleeds, specifically with those located in the lobar region, which may reflect subclinical cerebral amyloid angiopathy. It has been suggested that prolonged decreased concentrations of haemoglobin may contribute to microvascular damage [33] and that they exacerbate cerebral ischemia by creating a state of chronic cerebral hypoxia [34]. This hypoxic state increases amyloid- $\beta$ 1-42 levels via upregulation of $\beta$-secretase cleavage of amyloid precursor protein and $\beta$-secretase enzyme $[35,36]$, which promote amyloid plaque formation and contribute to neuronal death. In addition, cerebral microvascular smooth muscle cell damage resulting from hypoxic conditions further enhances amyloid angiopathy [37] and exacerbates chronic cerebral hypoxia [38].

With regard to neurodegenerative markers, only one previous study reported an association between decreased haemoglobin levels and cortical thinning in the frontal and parietal-temporal-occipital lobes in women [14]. Our results support previous findings by reporting associations between decreased haemoglobin levels and occipital cortical thinning. Furthermore, the effect estimates observed are consistent in both studies for the occipital lobe $(\beta=-0.011 \mathrm{~mm} ; 95 \% \mathrm{CI},-0.019$ to $0.004 \mathrm{~mm}$; compared with $\beta=-0.011 \mathrm{~mm} ; 95 \% \mathrm{CI},-$ 0.019 to $-0.003 \mathrm{~mm}$ ) [14]. The chronic hypoxic state induced by decreased haemoglobin levels may accelerate cortical thinning and hence neurodegeneration. Moreover, as indicated above, chronic hypoxia may induce amyloidogenic processing, leading to brain atrophy. 


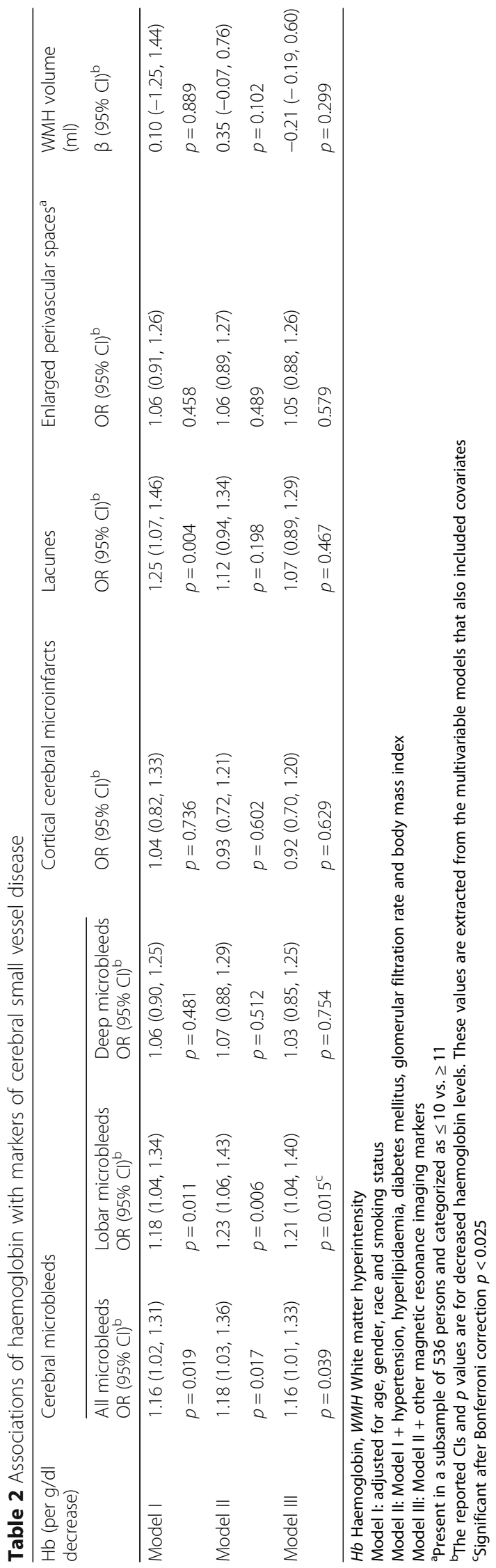




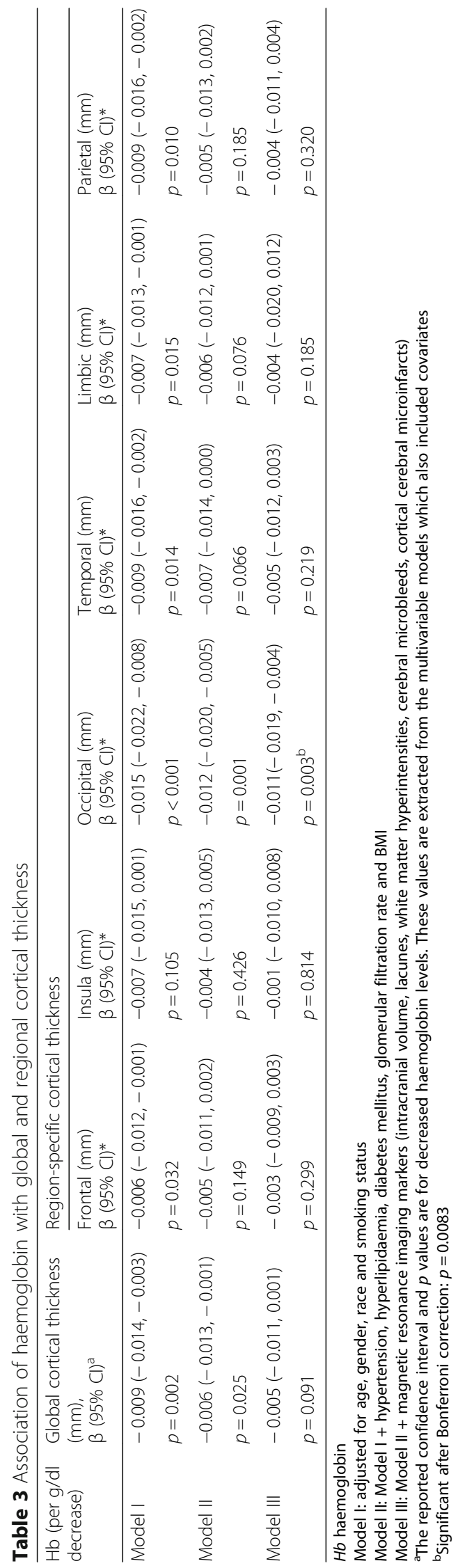




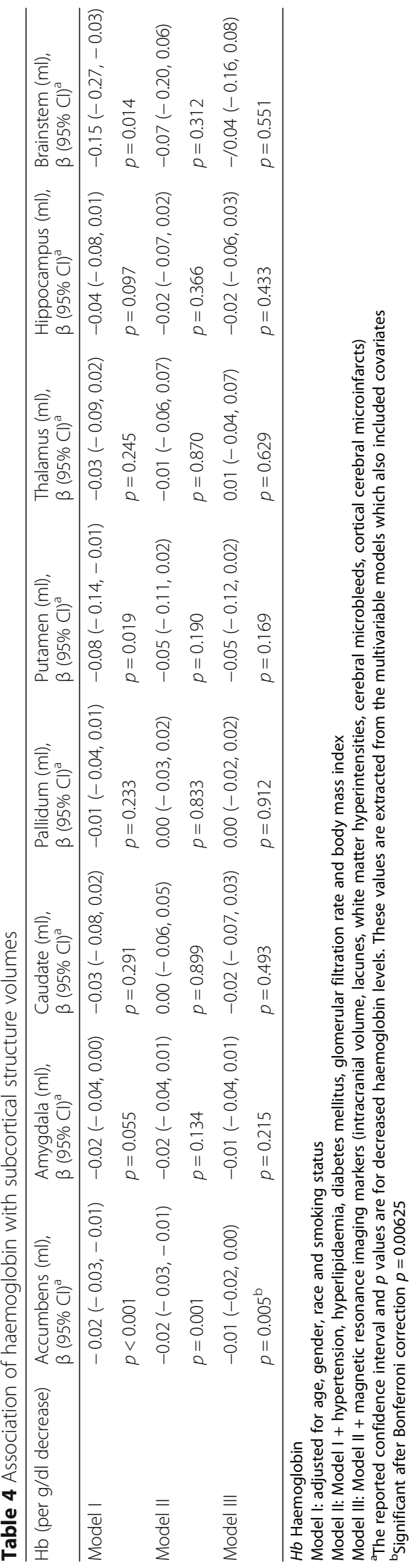




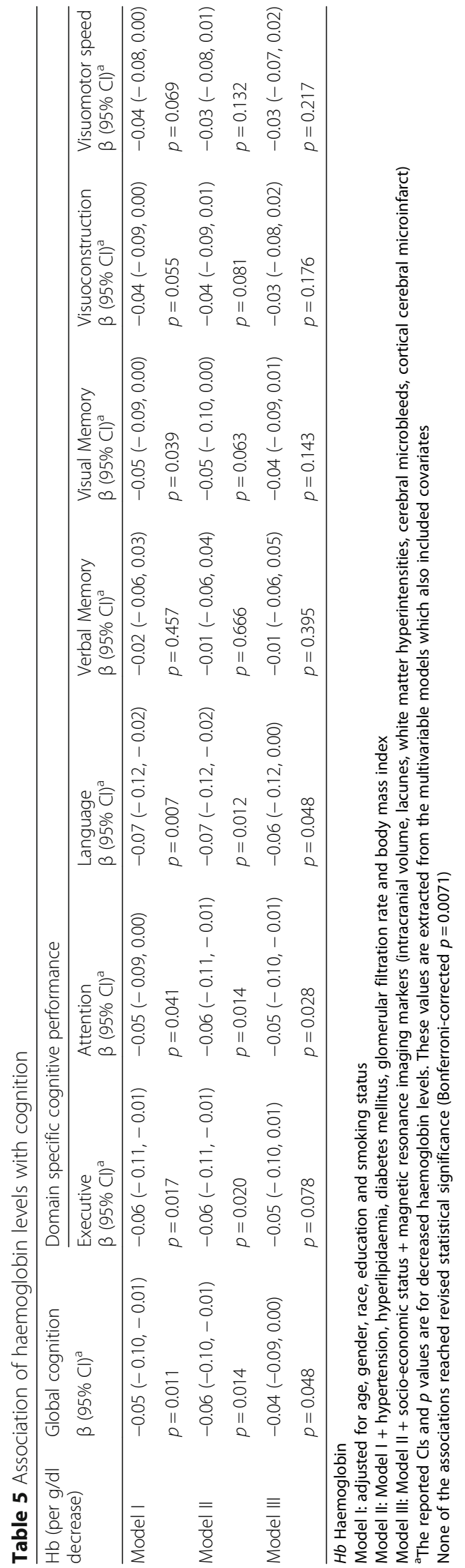


Interestingly, our study showed a consistent pattern in the association of decreased haemoglobin with microbleeds as well as with occipital thinning. Of note, the occipital cortex has the largest accumulation of cerebral amyloid angiopathy-related pathology on brain autopsy $[39,40]$. The occipital lobe is susceptible to neurotoxic effects of amyloid [41, 42], and the extent of amyloid burden correlated with the severity of cortical thinning and metabolism in these regions [43]. Taken together, a chronic hypoxic state could contribute to vascular amyloid formation selectively in the occipital lobe as compared with other lobes. Concomitantly, we also observed a significant association of decreased haemoglobin with smaller accumbens volumes. The accumbens is more susceptible than other subcortical structures to amyloid deposition [44], and thus its vulnerability to hypoxia-mediated amyloid insults is increased.

Our study also found an association between decreased haemoglobin levels and poorer cognitive function specifically in the domains of attention and language. It has been suggested that cerebral hypoxia or reduced aerobic capacity as a consequence of chronic low haemoglobin levels may contribute to cognitive decline. It has also been suggested that the accumbens nucleus is more vulnerable to amyloid and tau deposition. Moreover, it is reported that the accumbens is directly connected to the medial temporal lobe, cingulate gyrus and precuneus, which are associated with language and learning and hence impairments in these domains. This is in line with a previous study which showed a link between decreased haemoglobin levels and verbal fluency, a task assessing language [6]. However, these associations in our study did not reach the revised level of significance after applying the Bonferroni correction. Nevertheless, the direction of the effect estimates does suggest that there is a link between decreased lower haemoglobin levels and cognitive dysfunction. It is noteworthy that most of the individuals in this study were cognitively normal or in the preclinical stages of dementia, where mild to moderate decrease in haemoglobin concentrations may have less effect on oxygen delivery to the brain through compensatory mechanisms such as vascular dilation to maintain cerebral blood flow and hence produce less deleterious effects on cognition. It is also possible that decreased haemoglobin levels may simply be a marker of chronic inflammation, frailty and declining health status, the conditions associated with cognitive impairment and dementia. Similarly, CSVD is associated with underlying neuroinflammatory response, and could reflect global inflammatory status [45, 46], which in turn could result in lower haemoglobin levels. However, the interactions between CSVD and haemoglobin remain poorly investigated and should be addressed in future studies.
Our results suggest that decreased haemoglobin levels may lead to measurable brain parenchymal damage. Hence, persons with low haemoglobin levels might benefit from iron, folate and vitamin $B_{12}$ supplementation to improve cognitive performance. Although there have been previous clinical trials on exploring the beneficial effects of iron $[47,48]$, folate [49] and vitamin $B_{12}$ [50-52] on reducing cognitive impairment, these studies have not yielded promising results. However, it remains possible that a subgroup of the population with anaemia could benefit from vitamin B or iron supplementation. This has not been investigated to date in post hoc analyses of these trials, and it could be a potential area for further research.

Strengths of our study include that it is a large multi-ethnic Asian population-based study; standardized and automated imaging techniques were used to measure cortical thickness, volumes of white matter hyperintensity and subcortical structures; and extensive neuropsychological assessment. Limitations of the study include the following:

1. Forty-nine percent of screened-positive subjects were not included in the second phase of the study. Compared with the included subjects, excluded subjects were older, more likely to be female, to be less educated and to have hypertension and less likely to have hyperlipidaemia, which might suggest that these excluded subjects were more likely to be cognitively impaired. Despite this non-participation, we found significant associations between decreased levels of haemoglobin and MRI markers and cognitive impairment, suggesting that the true effect estimates must have been larger.

2. Owing to the cross-sectional design of our study, we were unable to examine the temporal associations of haemoglobin with development of cognitive impairment, and incident MRI.

3. Microbleeds as detected on MRI may provide an indirect measure of amyloid deposition, and other imaging modalities such as amyloid positron emission tomography could allow more accurate measurement of total (vascular and parenchymal) amyloid deposition in the brain.

4. We did not exclude or adjust for several other chronic conditions, such as chronic kidney disease, or immunocompromised diseases, such as cancer or human immunodeficiency virus, which may have the potential to decrease haemoglobin levels in blood. However, because this is a subsample of a population-based study, the influence of such diseases is estimated to be minimal. 


\section{Conclusions}

Our study showed significant associations of decreased haemoglobin levels with lobar microbleeds, cortical thinning, accumbens atrophy and cognitive impairment in a large multi-ethnic Asian cohort. Because modest reduction in haemoglobin may be able to induce subtle brain changes and hence cognitive impairment, future studies should ascertain whether iron, folate or $\mathrm{B}_{12}$ supplementation is able to ameliorate the onset and progression of anaemia-associated cognitive impairment and dementia.

\section{Abbreviations}

AMT: Abbreviated Mental Test; BMI: Body mass index; CSVD: Cerebral small vessel disease; eGFR: Estimated glomerular filtration rate; FLAIR: Fluidattenuated inversion recovery; MRI: Magnetic resonance imaging; WMSR: Wechsler Memory Scale-Revised

\section{Acknowledgements}

Not applicable.

\section{Funding}

The Epidemiology of Dementia in Singapore study is supported by the National Medical Research Council (NMRC), Singapore (NMRC/CG/NUHS/2010 grant nos. R-184-006-184-511 and NMRC/CSA/038/2013).

\section{Availability of data and materials}

The dataset used and analysed during the current study is available from the corresponding author upon reasonable request.

\section{Authors' contributions}

BT performed statistical analysis and drafting and revision of the manuscript. NV, HV, CYC and TYW participated in data acquisition and revised the manuscript for intellectual content. CC was responsible for the study concept and design, obtaining funding, and drafting and revising the manuscript. SH was responsible for the study concept and design and data acquisition, performed statistical analysis, and drafted and revised the manuscript. All authors read and approved the final manuscript.

\section{Ethics approval and consent to participate}

This study was conducted in accordance with the Declaration of Helsinki, and written informed consent was obtained from both the patients and the patients' caregivers. Ethics approval was granted by the National Healthcare Group Review Board of Singapore (NUHS1288/2010).

\section{Consent for publication}

Not applicable.

\section{Competing interests}

The authors declare that they have no competing interests.

\section{Publisher's Note}

Springer Nature remains neutral with regard to jurisdictional claims in published maps and institutional affiliations.

\section{Author details}

${ }^{1}$ Memory Ageing and Cognition Centre (MACC), National University Health System, Singapore, Singapore. ${ }^{2}$ Raffles Neuroscience Centre, Raffles Hospital, Singapore, Singapore. ${ }^{3}$ Departments of Radiology \& Medical Informatics, Erasmus University Medical Centre, Rotterdam, The Netherlands. ${ }^{4}$ Singapore Eye Research Institute, Singapore National Eye Centre, Singapore, Singapore ${ }^{5}$ Academic Medicine Research Institute, Duke-NUS Graduate Medical School, Singapore, Singapore. 'Department of Pharmacology, Yong Loo Lin School of Medicine, National University of Singapore, Level 4, Block MD3, 16 Medical Drive, Singapore 117600, Singapore. ${ }^{7}$ Departments of Epidemiology and Radiology and Nuclear Medicine, Erasmus University Medical Centre, Rotterdam, the Netherlands.
Received: 3 July 2018 Accepted: 11 October 2018

Published online: 06 November 2018

\section{References}

1. Patel KV. Epidemiology of anemia in older adults. Semin Hematol. 2008; 45:210-7

2. Bianchi VE. Role of nutrition on anemia in elderly. Clin Nutr ESPEN. 2016;11: e1-e11.

3. Chaves PH, Semba RD, Leng SX, Woodman RC, Ferrucci L, Guralnik JM, et al. Impact of anemia and cardiovascular disease on frailty status of communitydwelling older women: the Women's Health and Aging Studies I and II. J Gerontol A Biol Sci Med Sci. 2005;60:729-35.

4. Penninx BW, Guralnik JM, Onder G, Ferrucci L, Wallace RB, Pahor M. Anemia and decline in physical performance among older persons. Am J Med. 2003; 115:104-10.

5. Zakai NA, Katz R, Hirsch C, Shlipak MG, Chaves PH, Newman AB, et al. A prospective study of anemia status, hemoglobin concentration, and mortality in an elderly cohort: the Cardiovascular Health Study. Arch Intern Med. 2005;165:2214-20.

6. Shah RC, Wilson RS, Tang Y, Dong X, Murray A, Bennett DA. Relation of hemoglobin to level of cognitive function in older persons. Neuroepidemiology. 2009;32:40-6.

7. Jeong SM, Shin DW, Lee JE, Hyeon JH, Lee J, Kim S. Anemia is associated with incidence of dementia: a national health screening study in Korea involving 37,900 persons. Alzheimers Res Ther. 2017;9:94.

8. Beard CM, Kokmen E, O'Brien PC, Ania BJ, Melton LJ 3rd. Risk of Alzheimer's disease among elderly patients with anemia: population-based investigations in Olmsted County, Minnesota. Ann Epidemiol. 1997;7:219-24.

9. Atti AR, Palmer K, Volpato S, Zuliani G, Winblad B, Fratiglioni L. Anaemia increases the risk of dementia in cognitively intact elderly. Neurobiol Aging. 2006;27:278-84

10. Kurella Tamura M, Wadley VG, Newsome BB, Zakai NA, McClure LA, Howard $\mathrm{G}$, et al. Hemoglobin concentration and cognitive impairment in the renal REasons for Geographic And Racial Differences in Stroke (REGARDS) Study. J Gerontol A Biol Sci Med Sci. 2010;65:1380-6.

11. Son SJ, Lee KS, Na DL, Seo SW, Kim CH, Kim JH, et al. The effect of anemia and white matter hyperintensities (WMH) on cognitive impairment in patients with amnestic mild cognitive impairment (MCl). Arch Gerontol Geriatr. 2012;55:251-6.

12. Milward EA, Grayson DA, Creasey H, Janu MR, Brooks WS, Broe GA. Evidence for association of anaemia with vascular dementia. Neuroreport. 1999:10: 2377-81.

13. Inzitari M, Studenski S, Rosano C, Zakai NA, Longstreth WT Jr, Cushman M, et al. Anemia is associated with the progression of white matter disease in older adults with high blood pressure: the Cardiovascular Health Study. J Am Geriatr Soc. 2008;56:1867-72.

14. Park SE, Kim H, Lee J, Lee NK, Hwang JW, Yang JJ, et al. Decreased hemoglobin levels, cerebral small-vessel disease, and cortical atrophy: among cognitively normal elderly women and men. Int Psychogeriatr. 2016; 28:147-56.

15. Hilal S, Ikram MK, Saini M, Tan CS, Catindig JA, Dong YH, et al. Prevalence of cognitive impairment in Chinese: Epidemiology of Dementia in Singapore study. J Neurol Neurosurg Psychiatry. 2013;84:686-92.

16. Wardlaw JM, Smith EE, Biessels GJ, Cordonnier C, Fazekas F, Frayne R, et al. Neuroimaging standards for research into small vessel disease and its contribution to ageing and neurodegeneration. Lancet Neurol. 2013;12:822-38

17. Hilal S, Chai YL, van Veluw S, Shaik MA, Ikram MK, Venketasubramanian N, et al. Association between subclinical cardiac biomarkers and clinically manifest cardiac diseases with cortical cerebral microinfarcts. JAMA Neurol. 2017:74:403-10.

18. Cordonnier C, Potter GM, Jackson CA, Doubal F, Keir S, Sudlow CL, et al. improving interrater agreement about brain microbleeds: development of the Brain Observer MicroBleed Scale (BOMBS). Stroke. 2009;40:94-9.

19. Potter GM, Doubal FN, Jackson CA, Chappell FM, Sudlow CL, Dennis MS, et al. Counting cavitating lacunes underestimates the burden of lacunar infarction. Stroke. 2010;41:267-72.

20. Hilal S, Xin X, Ang SL, Tan CS, Venketasubramanian N, Niessen WJ, et al. Risk factors and consequences of cortical thickness in an Asian population. Medicine. 2015;94:e852. 
21. Hilal S, Amin SM, Venketasubramanian N, Niessen WJ, Vrooman H, Wong TY, et al. Subcortical Atrophy in cognitive impairment and dementia. J Alzheimers Dis. 2015;48:813-23.

22. Dubois B, Slachevsky A, Litvan I, et al. The FAB: a Frontal Assessment Battery at bedside. Neurology. 2000;55:1621-6.

23. Porteus SD. The Maze Test and clinical psychology. Palo Alto: Pacific Books; 1959.

24. Wechsler D. Wechsler Memory Scale - Revised. 3rd ed. San Antonio: Psychological Corp.; 1997.

25. Lewis RF, Rennick PM. Manual for the Repeatable Cognitive-PerceptualMotor Battery. Grosse Point Park: Axon Publishing; 1979.

26. Mack WJ, Freed DM, Williams BW, Henderson W. Boston Naming Test: shortened versions for use in Alzheimer's disease. J Gerontol. 1992:47:154-8.

27. Isaacs B, Kennie AT. The Set test as an aid to the detection of dementia in old people. Br J Psychiatry. 1973;123:467-70.

28. Smith A. Symbol Digit Modalities Test. Torrance: WPS; 1973.

29. Sahadevan S, Tan NJ, Tan T, et al. Cognitive testing of elderly Chinese people in Singapore: influence of education and age on normative scores. Age Ageing. 1997:26:481-6.

30. Diller L, Ben-Yishay Y, Gerstman LJ. Studies in cognition and rehabilitation in hemiplegia. New York: New York University Medical Center Institute of Rehabilitation Medicine; 1974.

31. Sunderland T, Hill JL, Mellow AM, et al. Clock drawing in Alzheimer's disease: a novel measure of dementia severity. J Am Geriatr Soc. 1989;37: 725-9.

32. Wechsler D. Wechsler Adult Intelligence Scale - Revised. San Antonio: Psychological Corp.; 1981.

33. Borzage MT, Bush AM, Choi S, Nederveen AJ, Vaclavu L, Coates TD, et al. Predictors of cerebral blood flow in patients with and without anemia. Appl Physiol. 2016:120:976-81.

34. Hare GM. Anaemia and the brain. Curr Opin Anaesthesiol. 2004;17:363-9.

35. Shiota S, Takekawa H, Matsumoto SE, Takeda K, Nurwidya F, Yoshioka Y, et al. Chronic intermittent hypoxia/reoxygenation facilitate amyloid- $\beta$ generation in mice. J Alzheimers Dis. 2013:37:325-33.

36. Sun X, He G, Qing H, Zhou W, Dobie F, Cai F, et al. Hypoxia facilitates Alzheimer's disease pathogenesis by up-regulating BACE1 gene expression. Proc Natl Acad Sci U S A. 2006;103:18727-32.

37. Auerbach ID, Vinters HV. Effects of anoxia and hypoxia on amyloid precursor protein processing in cerebral microvascular smooth muscle cells. J Neuropathol Exp Neurol. 2006;65:610-20.

38. Thomas T, Miners S, Love S. Post-mortem assessment of hypoperfusion of cerebral cortex in Alzheimer's disease and vascular dementia. Brain. 2015; 138:1059-69.

39. Thal DR, Ghebremedhin E, Rub U, Yamaguchi H, Del Tredici K, Braak H. Two types of sporadic cerebral amyloid angiopathy. J Neuropathol Exp Neurol. 2002;61:282-93.

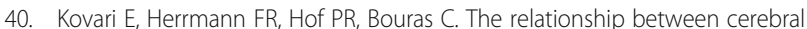
amyloid angiopathy and cortical microinfarcts in brain ageing and Alzheimer's disease. Neuropathol Appl Neurobiol. 2013;39:498-509.

41. Lewis DA, Campbell MJ, Terry RD, Morrison JH. Laminar and regional distributions of neurofibrillary tangles and neuritic plaques in Alzheimer's disease: a quantitative study of visual and auditory cortices. J Neurosci. 1987;7:1799-808.

42. Hof PR, Morrison JH. Quantitative analysis of a vulnerable subset of pyramidal neurons in Alzheimer's disease: II. Primary and secondary visual cortex. J Comp Neurol. 1990;301:55-64.

43. Buckner RL, Snyder AZ, Shannon BJ, LaRossa G, Sachs R, Fotenos AF, et al. Molecular, structural, and functional characterization of Alzheimer's disease: evidence for a relationship between default activity, amyloid, and memory. J Neurosci. 2005:25:7709-17.

44. Brilliant MJ, Elble RJ, Ghobrial M, Struble RG. The distribution of amyloid $\beta$ protein deposition in the corpus striatum of patients with Alzheimer's disease. Neuropathol Appl Neurobiol. 1997;23:322-5.

45. Rouhl RP, Damoiseaux JG, Lodder J, Theunissen RO, Knottnerus IL, Staals J, et al. Vascular inflammation in cerebral small vessel disease. Neurobiol Aging. 2012;33:1800-6.

46. Fu Y, Yan Y. Emerging role of immunity in cerebral small vessel disease. Front Immunol. 2018;9:67.

47. Milward EA, Bruce DG, Knuiman MW, Divitini ML, Cole M, Inderjeeth CA, et al. A cross-sectional community study of serum iron measures and cognitive status in older adults. J Alzheimers Dis. 2010;20:617-23.
48. Schiepers OJ, van Boxtel MP, de Groot RH, Jolles J, de Kort WL, Swinkels DW, et al. Serum iron parameters, HFE C282Y genotype, and cognitive performance in older adults: results from the FACIT study. J Gerontol A Biol Sci Med Sci. 2010;65:1312-21.

49. Ma F, Wu T, Zhao J, Song A, Liu H, Xu W, et al. Folic acid supplementation improves cognitive function by reducing the levels of peripheral inflammatory cytokines in elderly Chinese subjects with MCl. Sci Rep. 2016; 6:37486.

50. Ting SKS, Earnest A, Li H, Hameed S, Chang HM, Chen CLH, et al. B vitamins and cognition in subjects with small vessel disease: a substudy of VITATOPS a randomized, placebo-controlled trial. J Neurol Sci. 2017;379:124-6.

51. Li MM, Yu JT, Wang HF, Jiang T, Wang J, Meng XF, et al. Efficacy of vitamins B supplementation on mild cognitive impairment and Alzheimer's disease: a systematic review and meta-analysis. Curr Alzheimer Res. 2014;11:844-52.

52. Hankey GJ, Ford AH, Yi Q, Eikelboom JW, Lees KR, Chen C, et al. Effect of B vitamins and lowering homocysteine on cognitive impairment in patients with previous stroke or transient ischemic attack: a prespecified secondary analysis of a randomized, placebo-controlled trial and meta-analysis. Stroke. 2013:44:2232-9.

\section{Ready to submit your research? Choose BMC and benefit from:}

- fast, convenient online submission

- thorough peer review by experienced researchers in your field

- rapid publication on acceptance

- support for research data, including large and complex data types

- gold Open Access which fosters wider collaboration and increased citations

- maximum visibility for your research: over $100 \mathrm{M}$ website views per year

At $\mathrm{BMC}$, research is always in progress.

Learn more biomedcentral.com/submissions 